\title{
Wavelet based Despeckling of Medical Ultrasound Images using Speckle Reducing Anisotropic Diffusion and Local Wiener Filtering
}

\author{
Manami Barthakur \\ Department of Electronics and Communication Engineering, \\ Tezpur University, \\ Tezpur, India \\ manamibarthakur@gmail.com
}

\author{
Deepika Hazarika \\ Department of Electronics and Communication Engineering, \\ Tezpur University, \\ Tezpur, India \\ deepika@tezu.ernet.in
}

\author{
Vijay Kumar Nath \\ Department of Electronics and Communication Engineering, \\ Tezpur University, \\ Tezpur, India \\ vknath@tezu.ernet.in
}

\begin{abstract}
Multiplicative speckle noise which is inherently present in medical ultrasound images degrades the important clinical informations and badly affects the quality of the diagnosis. It is necessary to reduce the speckle noise to improve the visual quality of ultrasound images for better diagnoses. In this paper, a wavelet based method for despeckling of the ultrasound images is introduced where a local Wiener filter along with speckle reducing anisotropic diffusion (SRAD) filter are employed in a homomorphic framework. The signal variance in the local wiener filter is estimated from the output image of the SRAD filter. Since the size and shape of the locally adaptive window is an important issue in estimating the signal variance, nearly arbitrarily shaped windows are used for better performance. The experimental results using synthetically speckled ultrasound images show that the speckle noise is reduced to a great extent while preserving the important clinical information. In order to demonstrate the effectiveness of the proposed method, the method is compared with several other existing methods in terms of peak signal to noise ratio (PSNR), structural similarity index (SSIM), edge preservation index $(\beta)$, and standard deviation to mean $(\mathrm{S} / \mathrm{M})$ ratio.
\end{abstract}

\section{General Terms:}

Computer Science, Image Processing

\section{Keywords:}

Ultrasound images, Speckle reduction, SRAD, Local Wiener filter, Wavelet, Homomorphic filtering

\section{INTRODUCTION}

Medical ultrasound images are usually contaminated with a kind of noise called speckle noise. The speckle noise degrades the quality of the medical ultrasound images significantly and therefore makes the discrimination of the fine details in the images for medical diagnostic examinations very difficult. It is necessary to reduce the speckle noise from the medical ultrasound images while preserving the important features in order to improve the visual quality for better diagnoses.

Several methods have been proposed in the literature for reducing speckle noise from medical ultrasound images. The conventional methods described in [10], [5] and [9] often oversmooths the important details while reducing the speckle noise. The adaptive weighted median filter (AWMF) proposed by Loupas et al. [11] also reduces speckle noise but the edges in the images also gets blurred in the process. In homomorphic wiener filtering framework discussed in [8], logarithmic transformation is used before the Wiener filter, in order to obtain a approximately speckle noise free image. Over the past few years, 2D discrete wavelet transforms (DWT) have been popularly used for medical image denoising. Pizurica et al. [14] used a shrinkage function in order to obtain the noise free wavelet coefficients of the medical ultrasound image. Many denoising algorithms in homomorphic framework have been proposed. Achim et al. [1] proposed a wavelet based method to estimate the noise free coefficients using a Bayesian estimator that employs the alpha-stable pdf. The authors in [17] combined homomorphic filtering principle along with elliptical thresholding concept in complex discrete wavelet transform domain. The authors in [7] employed a maximum a posteriori (MAP) estimator in wavelet domain to estimate the noise free coefficients. The authors used Rayleigh statistical distribution to model the magnitude of speckle in the log domain and Gaussian distribution to model the wavelet coefficients of log transformed reflectivity of medical ultrasound images. 
Few wavelet based noise filtering schemes often requires the estimation of signal variance in wavelet domain. Various methods are reported such as [2] and [4] where the signal variance is estimated using noisy neighborhood with square shaped windows. In [16] the authors proposed a concept of doubly local Wiener filtering algorithm to suppress additive white Gaussian noise where directional windows are used to estimate the signal variance. The authors, in their work used a pilot image which is a denoised image obtained by the first local wiener filter with directional window to estimate the signal variance. The estimated signal variance is used in the second local wiener filter which is applied to the noisy image. In this paper, a algorithm is proposed which uses both SRAD and wavelet domain local Wiener filtering to reduce speckle noise from ultrasound images in homomorphic framework. In wavelet domain local Wiener filtering, the quality of the signal variance estimation is highly important. Therefore, we use efficient SRAD filter [20] output as the pilot image to estimate the signal variance. The SRAD is a spatial filter which performs well both at the edges and homogeneous regions in speckled images. Hence the quality of the estimated signal variance is much better and the local wiener filter performs well to reduce the speckle from the ultrasound image.

The paper is organized as follows. In section II the review of SRAD filter and the arbitrarily shaped window based local wiener filter is presented. In section III the proposed algorithm is demonstrated. Then in section IV the results of our method is compared with some other methods of denoising. At last the conclusion is given in section $\mathrm{V}$.

\section{BRIEF OVERVIEW ON SPECKLE REDUCING ANISOTROPIC DIFFUSION (SRAD) AND LOCAL WIENER FILTER}

For speckle reduction, Yu and Acton [20] proposed an improved approach of anisotropic diffusion concept proposed by Perona and Malik [13]. The partial derivative equations is given by [20],

$$
\left\{\begin{array}{l}
\partial I(x, y ; t) / \partial t=\operatorname{div}[a(q) \nabla I(x, y ; t)] \\
I(x, y ; 0)=I_{0}(x, y),\left(\partial I(x, y ; t) /\left.\partial \vec{f}\right|_{\delta \omega}=0\right.
\end{array}\right.
$$

where $I_{0}(x, y)$ is the intensity image having finite power and no zero values over the image, $t$ is the diffusion time, $\delta \omega$ denotes the border of $\omega, \vec{f}$ is the outer normal to $\delta \omega$ given by [20],

$$
a(q)=\frac{1}{\left.1+\left[q^{2}(x, y ; t)-q_{0}^{2}(t)\right] / q_{0}^{2}(t)\left(1+q_{0}^{2}(t)\right)\right]}
$$

or

$$
a(q)=\exp -\left[q^{2}(x, y ; t)-q_{0}^{2}(t)\right] /\left[q_{0}^{2}(t)\left(1+q_{0}^{2}(t)\right)\right]
$$

where $\mathrm{q}(\mathrm{x}, \mathrm{y} ; \mathrm{t})$ represents the instantaneous coefficient of variation at position $(\mathrm{x}, \mathrm{y})$ which is given by [20],

$$
q(x, y ; t)=\sqrt{\frac{\frac{1}{2}\left(\frac{|\nabla I|}{I}\right)^{2}-\left(\frac{1}{4^{2}}\right)\left(\frac{\nabla^{2} I}{I}\right)^{2}}{\left[1+\left(\frac{1}{4}\right)\left(\frac{\nabla^{2} I}{I}\right)\right]^{2}}}
$$

and $q_{0}(t)$ is the scale factor of speckle and for ultrasonic images it is given by [20],

$$
q_{0}(t)=q_{0} \exp [-\psi t]
$$

where $\psi$ is a constant and $q_{0}$ is the speckle coefficient of variation in the input image.
The SRAD algorithm has shown very good results in the literature and exhibits excellent performance in terms of smoothing homogeneous regions and preserving edges compared to conventional anisotropic diffusion, Lee and frost filter.

\subsection{Local Wiener filtering using arbitrary shaped windows}

Michak et al. [12] proposed a locally adaptive window based maximum likelihood (LAWML) estimate for signal variance estimation. It is a low-complexity, but powerful method, which shows the dependency of the local wavelet coefficients within each scale. A wavelet domain noisy image can be modeled as,

$$
z=s+n
$$

where $\mathrm{z}$ is the noisy image wavelet coefficients, $\mathrm{s}$ are the original image coefficients in wavelet domain and $\mathrm{n}$ is assumed to be an independent and identically distributed white Gaussian noise with zero mean and variance $\hat{\sigma}_{n}^{2}$. To estimate s from noisy coefficients $\mathrm{z}$, the minimum mean square estimator of $s(m)$ is given by [12],

$$
\hat{s}(m)=\frac{\hat{\sigma}_{m}^{2}}{\hat{\sigma}_{m}^{2}+\hat{\sigma}_{n}^{2}} z(m)
$$

where $\hat{\sigma}_{m}^{2}$ is the signal variance. The signal variance estimator used in LAWML method is given by [12],

$$
\hat{\sigma}_{m}^{2}=\max \left(0, \frac{1}{|W|} \sum_{z_{i} \in W} z_{i}^{2}-{\sigma_{n}}^{2}\right)
$$

where $W$ is the number of coefficients in the local window. It is to be noted that the performance of the (7) depends highly on the quality of variance estimation. The LAWML focuses only on a fixed window size for the noisy neighborhood.

K. Eom and Y.S. Kim developed a wavelet based denoising scheme with nearly arbitrarily shaped windows [3]. Fig.1 shows various types of arbitrarily shaped windows. They assumed $g_{m, 0}, . . g_{m, P-1}$ to be the arbitrarily shaped windows. The signal variance for each wavelet coefficient is calculated using these arbitrarily shaped windows until the homogeneity of the variance is achieved. The measure of homogeneity is defined as the normalized difference of variances, that is [3],

$$
N_{m, p}=\frac{\left|\sigma_{m, p}^{2}-\sigma_{m, 0}^{2}\right|}{\sigma_{m, 0}^{2}}, p=0,1,2, \ldots . P-1
$$

where $\sigma_{m, p}^{2}$ is the local variance of the region $g_{m, p}$ which is approximately calculated by [3]

$$
\sigma_{m, p}^{2}=\frac{1}{\left|W_{p}\right|} \sum_{y_{i} \in W_{p}} y_{i}^{2}
$$

where $\left|W_{p}\right|$ is the number of coefficients and $W_{p}$ are the coefficients within $g_{m, p}$. The binary factor $b_{m, p}$ which indicates whether the variance $\sigma_{m, p}^{2}$ is homogeneous with $\sigma_{m, 0}^{2}$, or not, is defined as [3]

$$
b_{m, p}= \begin{cases}1, & \text { if } N_{m, p}<t h \\ 0, & \text { otherwise }\end{cases}
$$

where 'th' is the theshold which is defined as [3] 


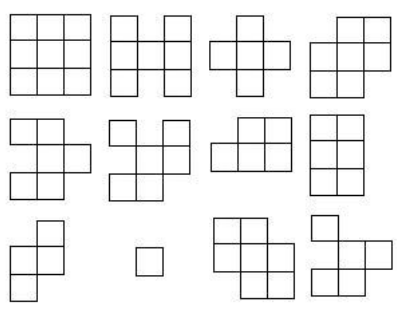

Fig. 1. Various types of arbitrarily shaped windows

$$
t h=\lambda 2^{(K-k)}, k=0, \ldots . K
$$

where, $\lambda$ is a scaling constant which was taken as $0.1, k=0$ for finest scale and $k=K$ for coarsest scale of the wavelet decomposition.Then the signal variance estimation is [3]

$$
\hat{\sigma}_{m}^{2}=\max \left(0, \frac{\sum_{p=0}^{P-1} \sigma_{m, p}^{2} \cdot b_{m, p}}{\sum_{p=0}^{P-1} b_{m, p}}-\sigma_{n}^{2}\right)
$$

Employing (7) with the above estimated signal variance, image denoising can be achieved.

\section{THE PROPOSED METHOD}

\subsection{The frame work:}

The speckle noise in the medical ultrasound image can be modeled as [8],

$$
r(m)=l(m) \eta_{m}+\eta_{a}
$$

where, $l(m)$ is the clean image, $r(m)$ is the noisy image and $\eta_{m}$ and $\eta_{a}$ are the multiplicative and additive noise respectively. The additive noise can be neglected as the effect of this noise is small, i.e $\eta_{a} \approx 0$. Therefore the equation can be written as,

$$
r(m)=l(m) \eta_{m}
$$

Logarithmic transformation is used to make this multiplicative noise into the additive one. i.e,

$$
\log r(m)=\log l(m)+\log \eta_{m}
$$

$\eta_{m}$ is assumed to be additive white Gaussian noise in this paper. The standard noise suppression techniques can be used on this logarithmically transformed image in order to reduce speckle noise. A denoised image can be obtained after applying denoising scheme and by applying the inverse of logarithmic transform. This framework of denoising is known as homomorphic filtering.

\subsection{The proposed algorithm:}

In this work, we use local Wiener filtering based algorithm in the homomorphic framework for denoising of ultrasound images. Since the performance of the filtering highly depends on the quality of the signal variance estimation, the estimation is obtained using a pilot image which is a denoised output by SRAD filter and calculated using arbitrarily shaped windows. The block diagram for the proposed method is given in the Figure 2 and the algorithm is given below.

Step 1: The noisy image is processed with SRAD to get the pilot image.
Table 1. No of iterations for different variances

\begin{tabular}{|c|c|}
\hline Variance & No. of iteration \\
\hline .04 & 6 \\
\hline .07 & 8 \\
\hline .11 & 10 \\
\hline .15 & 12 \\
\hline
\end{tabular}

Step 2: The logarithmic transform of the pilot image is taken.

Step 3: Apply 2D discrete wavelet transform (DWT) on the pilot image.

Step 4: After decomposition the signal variance is estimated using arbitrarily shaped window and calculated using (17).

$$
\hat{\sigma}_{m}^{2}=\frac{\sum_{p=0}^{P-1} \sigma_{m, p}^{2} \cdot b_{m, p}}{\sum_{p=0}^{P-1} b_{m, p}}
$$

There are $2^{P-1}$ differently shaped windows as obtained in [3].For the simplicity of computation we take nine such windows i.e $P=9$.

Step 5: The logarithmic transform of the noisy image is taken.

Step 6: The discrete wavelet transform (DWT) of the log transformed noisy image taken.

Step 7: The noisy wavelet coefficients are modified using equ. (7) according to the local wiener filtering algorithm as discussed in section II. Here the signal variance estimator $\hat{\sigma}_{m}^{2}$ is obtained from Step 3.

Step 8: After local wiener filtering of the noisy coefficients inverse discrete wavelet transform (IDWT) is carried out.

Step 9: The "adjust mean" is carried out after 2D IDWT. Since in wavelet denoising algorithms, the noise reduction is carried out only in the detail subbands and the approximation subband is not subjected to any change. In such cases it is important to correct the biased mean in the approximation subband as proposed in [19]. This biased mean is introduced by the logarithmic transform and it is corrected by subtracting the mean value of the logarithmic transformed speckle from the output image of "IDWT".

Step 10: The inverse logarithmic transform is taken after the "mean adjust" and the denoised image is obtained.

\section{EXPERIMENTAL RESULTS}

To compare the performance of proposed method with other denoising schemes such as median filter, Gaussian average filter, homomorphic wiener filter, frost filter, AWMF, and SRAD we have used two ultrasound images, which are of thyroid and liver. Since original noise free images are not available, the images are first processed with state of the art GenLink [14] method. The denoising software is obtained from "http://telin.ugent.be/ sanja/". The resulting images are considered to be the noise free images. After getting the noise free images the images are synthetically speckled with different variances.

For signal variance estimation the noisy images are first processed with SRAD filter. Different number of iterations is used for different noise variance which is given in the table no 1 .

The filtered image is considered as pilot image. The 2D DWT with five level decomposition is performed on the log transformed pilot image and the $\log$ transformed noisy image, with wavelet ' $\mathrm{db} 8$ '. The signal variance is calculated from coefficients of the pilot image using arbitrarily shaped windows in which for simplicity of calculation we take nine varying shaped windows, i.e. $P=9$. The 


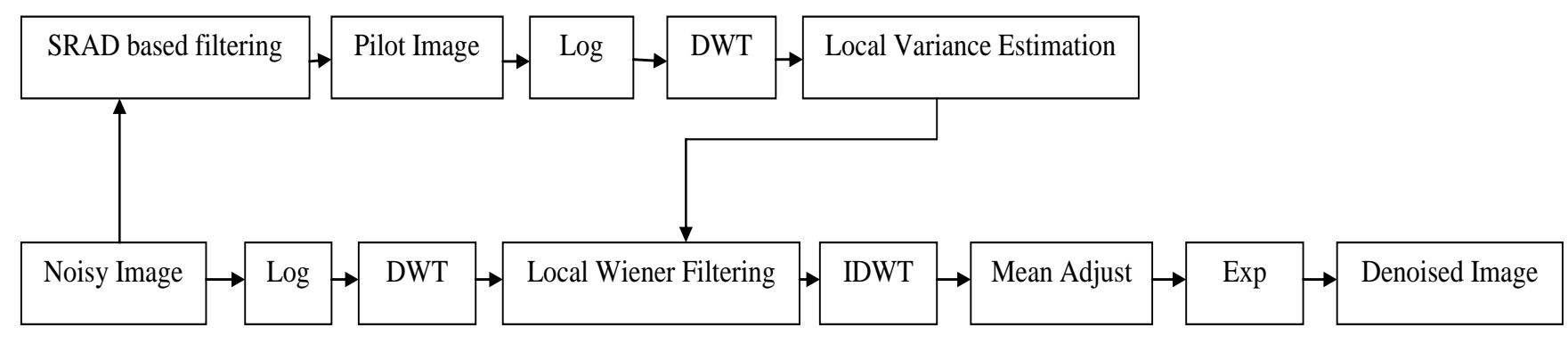

Fig. 2. Block diagram of proposed algorithm

proposed method is compared with other existing methods using the following metrics.

(1) Peak signal to noise ratio (PSNR): The PSNR is calculated using the following standard expression :

$$
P S N R=10 \log \left(\frac{255^{2}}{M S E}\right)
$$

Where,

$$
M S E=\frac{1}{X \times Y} \sum_{x=1}^{X} \sum_{y=1}^{Y}[I(x, y)-\hat{I}(x, y)]^{2}
$$

Where $I(x, y)$ and $\hat{I}(x, y)$ are the reference and filtered images respectively and $X \times Y$ is size of the image.

(2) Mean structural similarity index (MSSIM): The SSIM index [18] is a measure of similarity between two images and indicates perceived image quality. Higher the MSSIM between original and denoised image indicates better perceived image quality. For calculating the SSIM index, the matlab code, provided by the authors in their official website, is used.

(3) Edge preservation index ( $\beta$ ): It is a measure of edge preservation. For optimal edge preservation cases, $\beta$ should be close to unity. It is defined as [1],

$$
\beta=\frac{\Gamma(\Delta I-\overline{\Delta I}),(\hat{\Delta I}-\overline{\hat{\Delta I}})}{\sqrt{\Gamma(\Delta I-\overline{\Delta I}, \Delta I-\overline{\Delta I}) \cdot \Gamma(\hat{\Delta I-\hat{\Delta I}}, \hat{\Delta I-\hat{\Delta I}})}}
$$

where $\mathrm{I}$ and $\hat{I}$ are the clean and denoised image respectively. $\Delta I$ and $\hat{\Delta I}$ are the high pass-filtered versions of $\mathrm{I}$ and $\hat{I}$ respectively, obtained with a $3 \times 3$ pixel standard approximation of the Laplacian operator, the over line operator indicates the mean value, and

$$
\Gamma\left(I_{1}, I_{2}\right)=\sum_{i=1}^{K}\left(I_{1_{i}} . I_{2_{i}}\right)
$$

(4) $S / M$ ratio: It is a measure of speckle reduction performance [6].

(5) Coefficient of correlation (COC): It is a measure of noise suppression which is based on correlation [15]. It is defined as,

$$
C O C=\frac{\Gamma(I-\bar{I}),(\hat{I}-\overline{\hat{I}})}{\sqrt{\Gamma(I-\bar{I}, I-\bar{I}) \cdot \Gamma(\hat{I}-\overline{\hat{I}}, \hat{I}-\overline{\hat{I}})}}
$$

where $\mathrm{I}$ and $\hat{I}$ are the clean and denoised image respectively and the over line operator denotes the mean value, and

$$
\Gamma\left(I_{1}, I_{2}\right)=\sum_{i=1}^{K}\left(I_{1_{i}} . I_{2_{i}}\right)
$$

The simulated results are given in Table no. 2 and 3. In Figure 3 and Figure 4 the output images obtained from various other existing methods and proposed method, for synthetically speckled Liver ultrasound image (variance $=0.11$ ) and synthetically speckled Thyroid ultrasound image (variance $=0.11$ ) respectively, is shown. From the tables it is observed that the proposed method outperforms all other methods in terms of PSNR, S/M ratio, SSIM and $\beta$ for all the tested ultrasound images. The proposed method reduces the speckle noise very efficiently which is evident from the S/M ratio results and the edges are well preserved in the output images as compared to other schemes including SRAD filter which can be verified from the $\beta$ values.

\section{CONCLUSION}

In this paper, a wavelet domain local Wiener filtering based algorithm in a homomorphic framework is proposed where the signal variance is calculated from the SRAD filter output. The proposed method takes the advantage of excellent despeckling capability of SRAD filter to improve the final despeckling performance of wavelet domain local Wiener filter. We have tested our method with five different synthetically speckled ultrasound images and with different noise variance. The experimental results show that the proposed method performs better than all other existing methods including SRAD filter in terms of PSNR, SSIM index, S/M ratio and $\beta$ results.

\section{REFERENCES}

[1] A. Achim, A. Bezerianos, and P. Tsakalides. Novel bayesian multiscale method for speckle removal in medical ultrasound images. IEEE Trans. Medical Imaging, 20(8):772-783, Aug. 2001.

[2] S. G Chang, B. Yu, and M. Vetterli. Spatially adaptive wavelet thresholding with context modeling for image denoising. IEEE Trans. Image Processing, 9(9):1522-1531, 2000. 


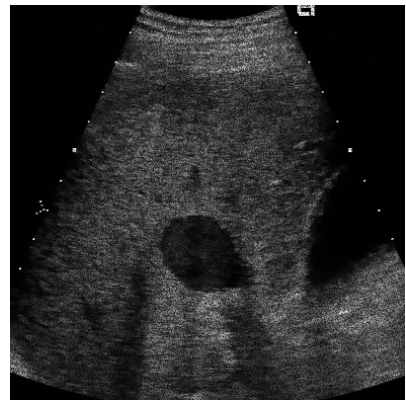

(a)

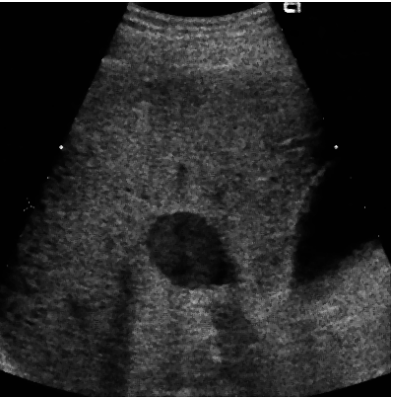

(b)

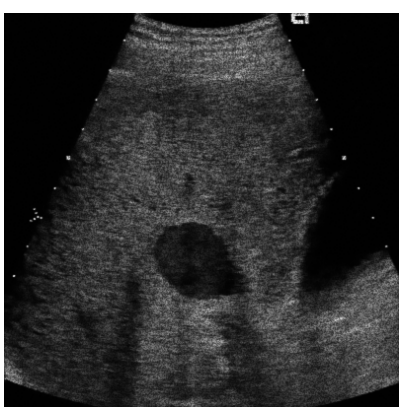

(c)

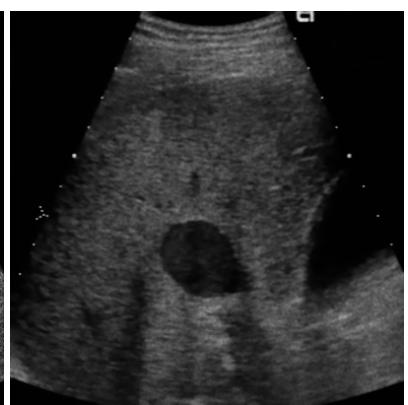

(d)

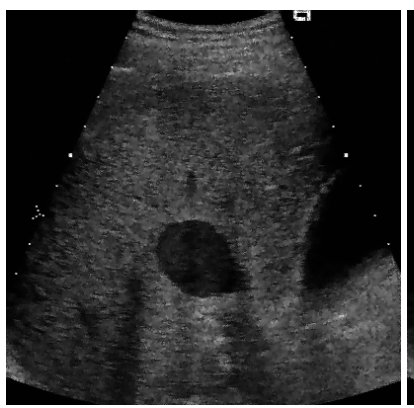

(e)

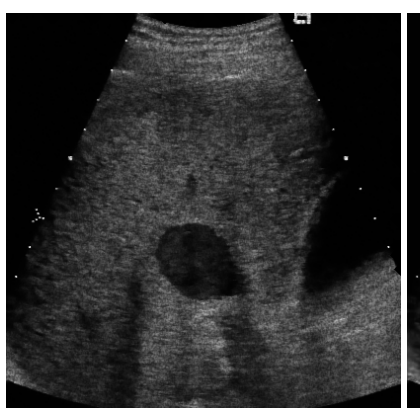

(g)

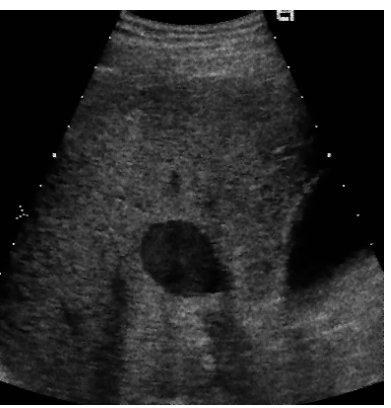

(f)

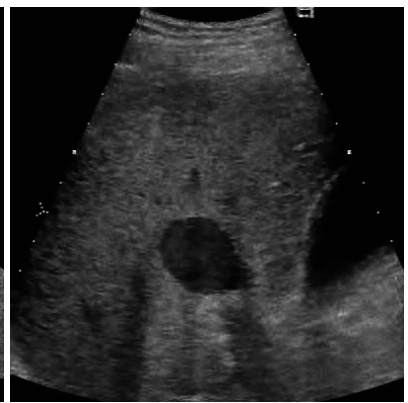

(h)

Fig. 3. Results of various despeckling methods for liver ultrasound image (a) Noisy Image (b) Median filter (c) Gaussian Average filter (d) Frost filter (e) AWMF filter (f) Wiener filter (g) SRAD filter(h) Proposed

[3] I. K. Eom and Y. S. Kim. Wavelet-based denoising with nearly arbitrarily shaped windows. IEEE Signal Process. Lett., 11(12):937-940, Dec. 2004.

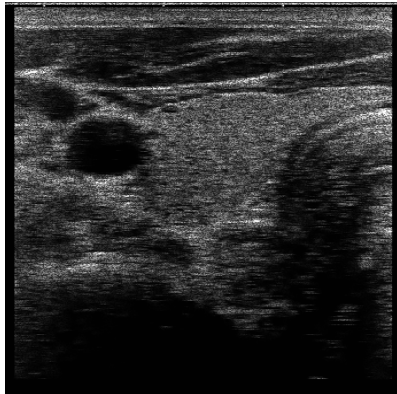

(a)

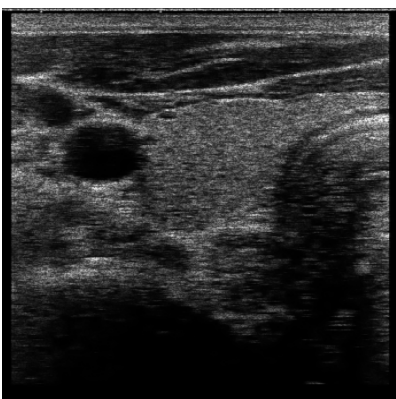

(c)

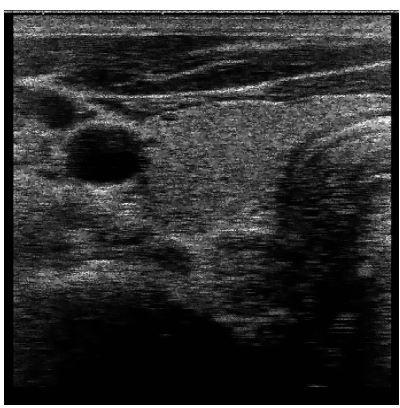

(e)

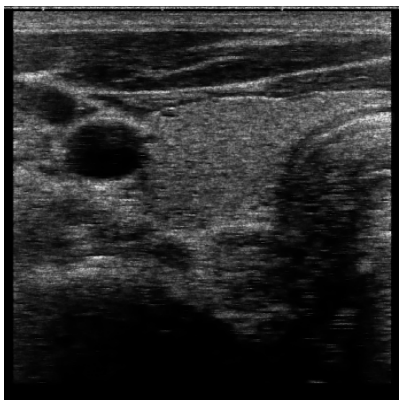

(g)

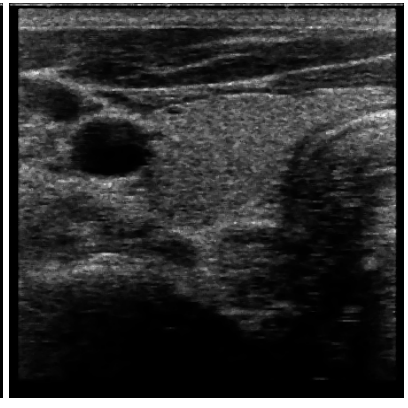

(b)

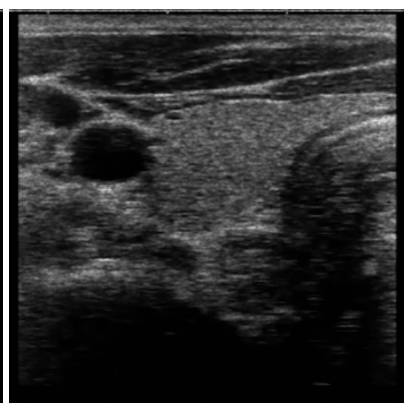

(d)

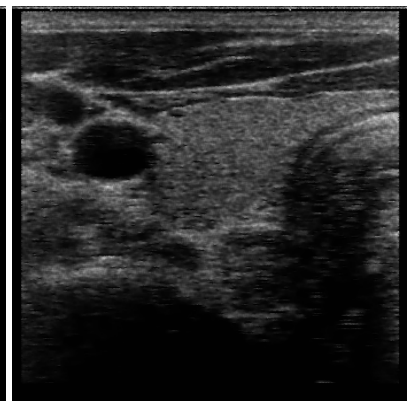

(f)

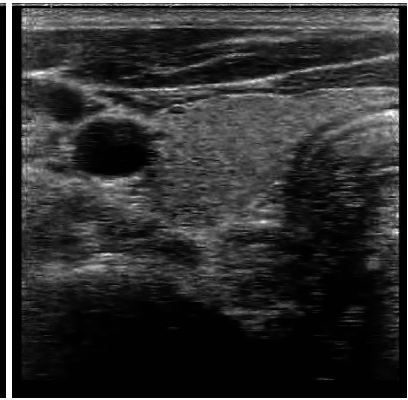

(h)
Fig. 4. Results of various despeckling methods for thyroid ultrasound image (a) Noisy Image (b) Median filter (c) Gaussian Average filter (d) Frost filter (e) AWMF filter (f) Wiener filter (g) SRAD filter(h) Proposed

[4] G. Fan and X. G. Xia. Novel bayesian multiscale method for speckle removal in medical ultrasound images. IEEE Signal Processing Letters, 8(5):125-128, May 2001. 
[5] V. S. Frost, J. A. Stiles, K. S. Shanmugan, and J. C. Holtzman. A model for radar images and its application to adaptive digital filtering of multiplicative noise. IEEE Trans. Pattern Analysis and Machine Intelligence, PAMI-4(2):157-165, 1982.

[6] D. Gnanadurai, V. Sadasivam, J. P. T. Nishandh, L. Muthukumaran, and C. Annamalai. Undecimated double density wavelet transform based speckle reduction in sar images. Computers and Electrical Engineering, 35:209-217, 2009.

[7] S. Gupta, R.C. Chauhan, and S.C. Saxena. Locally adaptive wavelet domain bayesian processor for denoising medical ultrasound images using speckle modelling based on rayleigh distribution,. IEE Proc.-Vis. Image Signal Processing, 152(1), Feb. 2005

[8] A. K. Jain. Fundamental of Digital Image Processing. Englewood Cliffs, NJ: Prentice-Hall, 1989.

[9] D. T. Kuan, A. A. Sawchuk, T. C. Strand, and P. Chavel. Adaptive noise smoothing fillter for images with signal dependent noise. IEEE Trans. Pattern Analysis and Machine Intelligence, 7(2):165-177, 1985.

[10] J. Lee. Digital image enhancement and noise filtering using local statistics. IEEE Transactions on Pattern Analysis and Mach. Intelligence, PAMI-2(2):165-168, Mar. 1980.

[11] T. Loupas, W. N. Mcdicken, and P. L. Allan. An adaptive weighted median fillter for speckle suppression in medical ultrasonic images,. IEEE Trans. Circuits and Systems, 36:129$135,1989$.

[12] M. K. Michak, I. Kozinsev, K. Ramchandran, and P. Moulin. Low-complexity image denoising based on statistical modeling of wavelet coeffcients. IEEE Signal Processing Letters, 6(12):300-303, Dec. 1999.

[13] P. Perona and J. Malik. Scale space and edge detection using anisotropic diffusion. IEEE Trans. Pattern Analysis and Machine Intelligence, 12:629-639, 1990.

[14] A. Pizurica, W. Philips, I. Lemahieu, and M. Acheroy. A versatile wavelet domain noise filtration technique for medical imaging. IEEE Trans. Medical Imaging, 22(3):323-331, 2003.

[15] F. Sattar, L. Floreby, G. Salomonsson, and B. Lovstrom. Image enhancement based on a nonlinear multiscale method. IEEE Trans Image Processing, 6:888-895, June 1997.

[16] Peng-Lang Shui. Image denoising algorithm via doubly local wiener filteltering with directional windows in wavelet domain. IEEE Signal Processing Letters, 12(10):681-684, Oct. 2005.

[17] S. Solbo and T. Eltoft. Speckle-noise reduction via rotated elliptical-thresholding in an homomorphic complexwavelet domain. IEEE Int. Conference on Image Processing, 3(4):585-588, Sept 2005.

[18] Z. Wang, A. Bovik, H. Sheikh, and E. Simoncelli. Image quality assessment: From error measurement to structural similarity. IEEE Trans. Image Processing, 13(4):600-612, Apr. 2004.

[19] H Xie, L. E. Pierce, and F. T. Ulaby. Sar speckle reduction using wavelet denoising and markov random field modeling. IEEE Trans. on Geoscience and Remote Sensing, 40:21962212, 2002.

[20] Yongjian Yu and Scott T. Acton. Speckle reducing anisotropic diffusion. IEEE Trans. Image Processing, 11(11):1260-1270, Nov. 2002. 
Table 2. Experimental results for thyroid ultrasound image

\begin{tabular}{|c|c|c|c|c|c|c|c|c|c|c|}
\hline \multirow[t]{2}{*}{ methods } & \multicolumn{5}{|c|}{$\sigma^{2}=.04$} & \multicolumn{5}{|c|}{$\sigma^{2}=.07$} \\
\hline & PSNR & MSSIM & $\beta$ & $\mathrm{S} / \mathrm{M}$ & $\mathrm{COC}$ & PSNR & MSSIM & $\beta$ & S/M & $\mathrm{COC}$ \\
\hline noisy & 26.95 & .8561 & .7035 & .7728 & .9611 & 24.51 & .7907 & .6208 & .7912 & .9346 \\
\hline Median & 27.06 & .8594 & .7045 & .7317 & .9698 & 26.10 & .7920 & .6214 & .7130 & .9372 \\
\hline $\begin{array}{c}\text { Gaussian } \\
\text { Average }\end{array}$ & 29.84 & .8639 & .7088 & .7341 & .9712 & 26.80 & .8164 & .6244 & .7411 & .9544 \\
\hline Frost & 28.30 & .8711 & .7112 & .6015 & .9765 & 27.61 & .8400 & .6288 & .6395 & .9641 \\
\hline AWMF & 28.96 & .8745 & .7125 & .5991 & .9791 & 27.82 & .8410 & .6297 & .6386 & .9678 \\
\hline wiener & 29.65 & .8872 & .7136 & .5987 & .9816 & 28.01 & .8598 & .6345 & .5887 & .9714 \\
\hline SRAD & 30.29 & .9042 & .7146 & .5945 & .9823 & 28.25 & .8618 & .6565 & .5787 & .9744 \\
\hline $\begin{array}{c}\text { Proposed } \\
\text { method }\end{array}$ & 30.53 & .9189 & .7142 & .5905 & .9837 & 28.75 & .8846 & .6386 & .5777 & .9759 \\
\hline \multirow[t]{2}{*}{ Methods } & \multicolumn{5}{|c|}{$\sigma^{2}=.11$} & \multicolumn{5}{|c|}{$\sigma^{2}=.15$} \\
\hline & PSNR & MSSIM & $\beta$ & S/M & $\mathrm{COC}$ & PSNR & MSSIM & $\beta$ & S/M & $\mathrm{COC}$ \\
\hline noisy & 22.56 & .7272 & .5459 & .7634 & .9026 & 21.26 & .6791 & .4966 & .6215 & .8739 \\
\hline Median & 25.17 & .7278 & .5468 & .7216 & .9145 & 24.37 & .6849 & .4968 & .6889 & .8862 \\
\hline $\begin{array}{l}\text { Gaussian } \\
\text { Average }\end{array}$ & 26.10 & .7421 & .5495 & .6831 & .9346 & 24.77 & .7192 & .4974 & .5837 & .9164 \\
\hline Frost & 26.93 & .7560 & .5543 & .5880 & .9535 & 25.09 & .7500 & .4999 & .5368 & .9464 \\
\hline AWMF & 27.06 & .7588 & .5549 & .5868 & .9572 & 25.47 & .7623 & .5016 & .5338 & .9476 \\
\hline wiener & 27.13 & .8166 & .5573 & .5814 & .9615 & 25.60 & .7715 & .5035 & .5281 & .9536 \\
\hline SRAD & 27.24 & .8245 & .5619 & .5714 & $\begin{array}{l}.9633 \\
\end{array}$ & 26.24 & .8167 & .5045 & .5046 & .9565 \\
\hline $\begin{array}{c}\text { Proposed } \\
\text { method }\end{array}$ & 27.52 & .8575 & .5638 & .5779 & .9675 & 26.67 & .8308 & .5041 & .4737 & .9594 \\
\hline
\end{tabular}

Table 3. Experimental results for liver ultrasound image

\begin{tabular}{|c|c|c|c|c|c|c|c|c|c|c|}
\hline \multirow[t]{2}{*}{ methods } & \multicolumn{5}{|c|}{$\sigma^{2}=.04$} & \multicolumn{5}{|c|}{$\sigma^{2}=.07$} \\
\hline & PSNR & MSSIM & $\beta$ & S/M & $\mathrm{COC}$ & PSNR & MSSIM & $\beta$ & S/M & $\mathrm{COC}$ \\
\hline noisy & 27.54 & .8013 & .4771 & .3561 & .9546 & 25.12 & .8496 & .3800 & .3891 & .9244 \\
\hline Median & 29.38 & .8908 & .4786 & .2785 & .9547 & 28.28 & .8505 & .3825 & .2670 & .9269 \\
\hline $\begin{array}{c}\text { Gaussian } \\
\text { Average }\end{array}$ & 30.91 & .8192 & .4799 & .3498 & .9579 & 28.43 & .8834 & .3856 & .3992 & .9349 \\
\hline Frost & 31.43 & .9321 & .4860 & .3135 & .9648 & 29.84 & .8990 & .3946 & .3539 & .9466 \\
\hline AWMF & 32.20 & .9345 & .4883 & .2965 & .9683 & 30.42 & 8965 & .3991 & .3102 & .9487 \\
\hline wiener & 32.43 & .9366 & .4909 & .2706 & .9622 & 30.99 & .9125 & .4145 & .2713 & .9564 \\
\hline SRAD & 32.94 & .9398 & .5409 & .2699 & .9725 & 31.27 & .9146 & .4199 & .2743 & .9610 \\
\hline $\begin{array}{l}\text { Proposed } \\
\text { method }\end{array}$ & 33.14 & .9412 & .5994 & .2690 & .9793 & 31.40 & .9174 & .5188 & .2612 & .9647 \\
\hline \multirow[t]{2}{*}{ Methods } & \multicolumn{5}{|c|}{$\sigma^{2}=.11$} & \multicolumn{5}{|c|}{$\sigma^{2}=.15$} \\
\hline & PSNR & MSSIM & $\beta$ & $\mathrm{S} / \mathrm{M}$ & $\mathrm{COC}$ & PSNR & MSSIM & $\beta$ & $\mathrm{S} / \mathrm{M}$ & $\mathrm{COC}$ \\
\hline noisy & 23.21 & .7916 & .3346 & .4519 & .8877 & 21.84 & .7503 & .2817 & .4868 & .8555 \\
\hline Median & 27.05 & .8074 & .3340 & .3171 & .8895 & 26.08 & .7663 & .2815 & .3086 & .8595 \\
\hline $\begin{array}{c}\text { Gaussian } \\
\text { Average }\end{array}$ & 27.79 & .8189 & .3356 & .4203 & .9064 & 26.54 & .8025 & .2832 & .4735 & .9268 \\
\hline Frost & 28.62 & .8558 & .3394 & .3660 & .9361 & .2811 & .8381 & .2851 & .2981 & .9356 \\
\hline AWMF & 28.95 & .8643 & .3410 & .3428 & .9466 & 28.54 & .8415 & .2961 & .2850 & .9365 \\
\hline wiener & 29.24 & .8736 & .3626 & .2813 & .9498 & 27.86 & .8401 & .3675 & .2656 & .9388 \\
\hline SRAD & 29.99 & .8816 & .3721 & .2647 & .9508 & 28.84 & .8528 & .4466 & .2598 & .9384 \\
\hline $\begin{array}{l}\text { Proposed } \\
\text { method }\end{array}$ & 30.29 & .8867 & .3958 & .2598 & .9551 & 29.11 & .8643 & .4881 & .2310 & .9397 \\
\hline
\end{tabular}

